\title{
Utilidad de la tomografía computarizada y la broncoscopia en los pacientes con hemoptisis. Análisis de 482 casos
}

\author{
M. HARO*, J. JIMÉNEZ, A. TORNERO, M. VIZCAYA, R. TIRADO, T. CROS \\ Sección de Neumología. Servicios de Medicina Interna y Radiología. Hospital General de \\ Albacete. *Hospital Universitario Dr. Josep Trueta. Girona
}

\begin{abstract}
COMPUTED CHEST TOMOGRAPHY AND BRONCHOSCOPY UTI LITY IN PATIENTS WITH HEMOPTYSIS. ANALYSIS OF 482 CASES
\end{abstract}

\section{RESUMEN}

Objetivo: Analizar la utilidad de la fibrobroncoscopia (FB) y la tomografía computarizada (TC) en el diagnóstico etiológico y la localización del sangrado en los pacientes con hemoptisis.

Métodos: Estudio descriptivo y prospectivo de la etiología o la localización del sangrado en 482 pacientes con hemoptisis a los que se les realizó una FB y una TC por la sospecha de una neoplasia, bronquiectasias o las características del sangrado. Se valoraron la edad, sexo, tabaquismo, características de la hemoptisis y los resultados de la radiografía de tórax, FB y TC.

Resultados: Edad 62 años (DE 13,6), 84,4\% varones, 80\% fumadores con un volumen de sangrado de 42,5 ml/día (DE 89) durante 15 días (DE 25). Las causas más frecuentes incluyeron las neoplasias (43\%), bronquiectasias (20\%), bronquitis crónica (14\%) y neumonías (8\%) junto a un 3\% de formas idiopáticas. La FB localizó el sangrado en el $77 \%$ y la TC identificó alteraciones compatibles con el posible origen del sangrado en el 83\%. En las neoplasias, la radiografía fue normal en el $11 \%$ y en el $87 \%$ la FB aportó el diagnóstico, mientras en 6 casos la TC fue normal. En ningún caso, la TC o la radiografía y la FB fueron normales simultánemente en las neoplasias. Cuando la radiografía era inespecífica (32\%), la TC aportó el diagnóstico etiológico en el $43 \%$ y la FB en el $14 \%$ ( $\mathrm{p}<0,001)$ o localizó el sangrado en el $52 \%$ la TC y el $23 \%$ la FB $(p<0,001)$.

Conclusiones: La FB y la TC fueron útiles y complementarias en el diagnóstico etiológico o localización del sangrado de nuestros pacientes. La FB fue la exploración más útil en los pacientes con una neoplasia y la TC de forma global o en los pacientes sin una neoplasia, especialmente si la radiografía era normal o inespecífica. El uso simultáneo de la TC o la radiografía y la FB evitó que ningún caso de neoplasia pasara desapercibida.

\begin{abstract}
Objective: We have examined the computed chest tomography $(C T)$ and bronchoscopy utility in diagnosis and accurately identifying the sites of pulmonary bleeding in patients with hemoptysis.

Methods: We prospectively reviewed the etiology and the site of ble eding of 482 patients with hemoptysis who had undergone bronchoscopy and CT when we suspected a carcinoma, bronchiectasis or the type of bleeding. We analyzed the age, sex, history of tobacco, bleeding, chest radiography, chest computed tomography and bronchoscopy.

Results: 62 years (DS 13.6), 84.4\% males, $80 \%$ smokers and a volu me of bleeding of 42,5 ml/day (DS 89) for 15 days (DS 25). Lung cancer was identified in $43 \%$, bronchiectasis in $20 \%$, chronic obstructive lung disease in $14 \%$, pneumonia $8 \%$ and an unknown etiology in only $3 \%$. Bronchoscopy located the site of bleeding in $77 \%$ and CT in $83 \%$. When we examine only the lung carcinomas, the chest radiography was normal in $11 \%$ and the bronchoscopy was diagnostic in 87\%, including 6 cases with a normal CT. Simultaneously chest CT or radiography and bron. choscopy detected all the lung cancers. When the chest radiography was inespecific (32\%), CT was diagnostic in $43 \%$ and bronchoscopy in $14 \%$ $(p<0.001)$ or localized the site of bleeding in 52\%with the CT or $23 \%$ with the bronchoscopy $(<0.001)$.

Conclusions: We concluded that bronchoscopy and CT were useful and complementary in etiologic diagnosis and to localize the site of blee ding in patients with hemoptysis. The bronchoscopy was preferable in patients with a lung cancer and the CT when we studied all the etiologies or the patients without a lung cancer, especially when the radiography was normal. When we simultaneously used a CT or a radiography and the bronchoscopy, all the lung cancers were detected.
\end{abstract}

KEY WORDS: Hemoptysis. Computed chest tomography. Bronchos copy.

PALABRAS CLAVE: Hemoptisis. Tomografía computarizada. Broncoscopia.

Haro M, Jiménez J, Tornero A, Vizcaya M, Tirado R, Cros T. Utilidad de la tomografía computarizada y la broncoscopia en los pacientes con hemoptisis. Análisis de 482 casos. An Med Interna (Madrid) 2002; 19: 59-65.

\section{INTRODUCCIÓN}

La hemoptisis es un síntoma alarmante de curso impredecible que puede ser la primera manifestación de un amplio espectro de enfermedades con una etiología, pronóstico o tratamiento variables (1-3). Una misma causa o prácticamente la mayoría de ellas pueden presentar un sangrado mínimo o amenazante y ser el aviso de una enfermedad grave o una hemorragia fatal que justifican la trascendencia del conocimiento de su etiología y la necesidad de utilizar distintas técnicas para llegar a un diagnóstico, localizarla e iniciar un tratamiento $(4,5)$.

Trabajo aceptado: 23 de octubre de 2001

Correspondencia: M. Haro Estarriol. Sección de Neumología (planta $4^{\mathrm{a}}$ B). Hospital Universitario Dr. Josep Trueta. Avda. de Francia s/n. 17007 Girona. e-mail: mip.mharo@htrueta.scs.es. 
Aunque la radiografía de tórax puede orientar o evidenciar anomalías sugestivas de una determinada etiología o localización, ésta no siempre es específica o es normal aconsejando la utilización de otras técnicas cuya indicación ha quedado establecida a partir de estudios retrospectivos o casos aislados en la mayoría de los consensos actuales (2,5). De todas las exploraciones analizadas en los pacientes con hemoptisis, la fibrobroncoscopia (FB) es considerada imprescindible por algunos autores y la tomografía computarizada (TC) podría complementarla o detectar lesiones pulmonares centrales y periféricas de una forma menos invasiva. Existe un debate no resuelto sobre la implantación rutinaria o selectiva de la TC y el uso generalizado de la FB, especialmente en los pacientes con una radiografía normal, cuyos criterios están directamente relacionados con las etiologías más frecuentes en cada medio y los criterios utilizados para justificar su realización (5-7). Así, ante la disponibilidad limitada de datos en nuestro medio hemos planteado como objetivo de este estudio la valoración y comparación de la utilidad de la FB y la TC en los pacientes con hemoptisis, prestando especial atención a aquellos casos con una radiografía de tórax normal y en los diagnosticados de una neoplasia.

\section{MATERIA Y MÉTODOS}

Estudio descriptivo y prospectivo de la etiología y la localización del sangrado de los pacientes remitidos por una hemoptisis a una consulta monográfica de acceso rápido de un área de referencia de aproximadamente 390.000 habitantes en el periodo comprendido entre Enero de 1996 y Noviembre de 1999 a los que se realizó una FB y una TC torácica de forma independiente durante los 20 días siguientes al sangrado. Para llegar a un diagnóstico etiológico, todos los pacientes iniciaron un mismo protocolo de estudio que incluía la realización de una historia clínica completa considerando la edad, sexo, tabaquismo, antecedentes personales y clínica, características de la hemoptisis (volumen diario y duración), radiografía de tórax (proyecciones posteroanterior y perfil), 3 baciloscopias de esputo, pruebas funcionales respiratorias (espirometría y gasometría arterial basal), hemograma y coagulación. Todos los casos fueron sometidos a una FB realizada por el mismo médico empleando como instrumental un broncoscopio Olympus modelo OES10 y OES P20D (Medical Europa S.A.). Esta exploración incluía la realización sistemática de un broncoaspirado (BAS) para el estudio de micobacterias (tinción de Ziehl-Neelsen y cultivo de Löwestein) y citología (tinción de Papanicolau), junto a biopsias u otras técnicas si eran necesarias ante la presencia de una lesión endobronquial sospechosa, tumores endoscópicamente visibles u otra sospecha etiológica. Los hallazgos de la FB fueron divididos en: a) exploración normal, b) lesión sospechosa de una neoplasia, c) bronquitis difusa, d) bronquitis localizada por su predominio en un determinado lóbulo o segmento pulmonar, e) presencia aislada de sangrado activo y f) restos hemáticos localizados solo en un determinado lóbulo o segmento. Si en una primera exploración se observaban coágulos endobronquiales con posibles funciones hemostáticas o riesgo de sangrado, no eran aspirados y se repetía la exploración en 7-10 días para descartar la presencia de lesiones subyacentes.

La TC fue realizada sin conocer los resultados de la FB a todos aquellos pacientes que cumplían alguno de los siguientes criterios: a) sospecha de una neoplasia pulmonar, b) sospe- cha clínica, radiológica o broncoscópica de bronquiectasias, c) una hemoptisis recidivante (episodios repetidos en un tiempo de 1-2 meses), d) persistencia del sangrado superior a los 10 días sin un diagnóstico claro o que lo justificara, e) un volumen de sangrado superior a $50 \mathrm{ml} /$ día, y f) para valorar anomalías poco definidas en la radiografía simple o en otras etiologías en las que pudiera ser de ayuda. Esta exploración fue realizada administrando contraste intravenoso con cortes de 1-2 mm cada $10 \mathrm{~mm}$ desde el estrecho torácico superior hasta las bases pulmonares, disminuyendo el intervalo a $5 \mathrm{~mm}$ en las bases o zonas sospechosas de alguna patología. Si existía la sospecha o confirmación de una neoplasia pulmonar ampliamos la exploración hasta incluir las glándulas suprarrenales y el hígado. Los resultados fueron evaluados independientemente por un radiológo y un neumólogo, cuyo acuerdo permitió clasificar a la exploración como normal o con alteraciones específicas relacionadas con la hemoptisis.

La etiología de la hemoptisis fue determinada tras la valoración conjunta de los hallazgos de la $\mathrm{FB}$, la TC y el resto de estudios realizados con un seguimiento mínimo de 17 meses para su confirmación en los pacientes sin una neoplasia. El diagnóstico de una neumonía o absceso pulmonar se basaba en la clínica, leucocitosis, radiografía y ausencia de lesiones endobronquiales en la FB o la TC con posterior seguimiento y resolución8. La presencia de dilataciones bronquiales periféricas con un diámetro transversal superior a la arteria adyacente fue catalogado de bronquiectasias (9). Las neoplasias pulmonares fueron confirmadas histológica y/o citológicamente a través de la FB y en caso negativo, por una punción aspirativa transtorácica o biopsias endoscópicas por fluoroscopia en las lesiones más periféricas. La tuberculosis fue diagnosticada mediante la confirmación microbiológica o la existencia de granulomas caseificantes en las biopsias, mientras que su ausencia y el antecedente de enfermedad asociada a lesiones residuales o cicatriciales fue valorado como etiología cuando la TC era compatible y en la FB se observaron restos hemáticos localizados, sangrado activo o habíamos excluido otras causas. Los aspergilomas fueron diagnosticados por toracotomía y cultivos específicos. El diagnóstico de una bronquitis crónica o asma bronquial se estableció cuando la historia clínica y las pruebas funcionales eran compatibles con los consensos actuales $(10,11)$. El diagnóstico de miocardiopatía o valvulopatía fue confirmado por la ecocardiografía.

Se realizó un análisis descriptivo de las etiologías y los hallazgos de la FB o la TC de forma independiente y comparativo entre todos los pacientes, en las causas más frecuentes, en los casos con radiografía normal y en los diagnosticados de neoplasia pulmonar. Los porcentajes de los hallazgos según las etiologías fueron comparados mediante la ji cuadrado o el test exacto de Fisher cuando fue necesario considerándolos estadísticamente significativos cuando la p era inferior a 0,05 .

\section{RESULTADOS}

\section{VALORACIÓN DE LA FB Y LA TC EN TODOS LOS PACIENTES}

De un total de 752 pacientes que acudieron a la consulta se realizó una TC torácica a $516(69 \%)$ por cumplir los criterios previamente descritos. De los 516 casos, fueron excluidos 34 $(6,6 \%)$ por no realizarse una FB y cuyos diagnósticos incluyeron a nueve casos de bronquiectasias, cuatro aneurismas de aorta 
complicados, cuatro casos con bronquitis crónica, tres tromboembolismos pulmonares, tres cardiopatías, dos cuerpos extraños y nueve que no aceptaron o colaboraron en su realización. Los 482 restantes $(93,4 \%)$ fueron motivo del estudio y presentaron una edad de 62 años (desviación estándar DE 13,6, entre 14 y 93 años), con 56 casos de menos de 45 años (12\%), 407 varones $(84,4 \%)$ y 385 fumadores $(80 \%)$ de 56 paquetes/año (DE 32,5). La hemoptisis fue de 42,5 ml/día (DE 89) con un volumen inferior a $10 \mathrm{ml} /$ día en 288 casos $(60 \%)$ o superior a $200 \mathrm{ml} /$ día en 19 (4\%) y una duración del sangrado de 15 días (DE 25). La etiología fue neoplásica en 208 casos (43\%) y benigna en 274 $(57 \%)(\mathrm{p}<0,001)$, cuya distribución queda reflejada en la tabla I. La miscelánea de los pacientes con una neoplasia incluyó a casos únicos con un tumor glómico traqueal, un carcinoma adenoide quístico de carina y un papiloma endobronquial. La miscelánea de los pacientes con etiologías no neoplásicas incluyó a dos casos con un cuerpo extraño, dos lupus eritematosos con afectación pulmonar, dos síndromes de Goodpasture, dos neumonías lipoideas, dos valvulopatías mitrales y casos únicos de hemosiderosis idiopática, granulomatosis de Wegener y bronquiolitis obliterante con neumonía organizada.

\begin{tabular}{|c|c|c|}
\hline \multicolumn{3}{|c|}{ TABLA I } \\
\hline \multicolumn{3}{|c|}{$\begin{array}{l}\text { DISTRIBUCIÓ N ETIO LÓ GICA DE LO S PACIENTES } \\
\text { CON HEM O PTISIS }\end{array}$} \\
\hline & Casos & $\%$ \\
\hline Neoplasias & 208 & 43 \\
\hline Carcinoma epidermoide & 109 & \\
\hline CPCP & 42 & \\
\hline Adenocarcinoma & 24 & \\
\hline Carcinoma indiferenciado & 12 & \\
\hline M etástasis & 8 & \\
\hline Neoplasia esófago & 4 & \\
\hline Carcinoma células grandes & 3 & \\
\hline Tumor carcinoide & 3 & \\
\hline M iscelánea & 3 & \\
\hline Bronquiectasias & 96 & 20 \\
\hline Bronquitis crónica & 68 & 14 \\
\hline Neumonía/Absceso & 37 & 8 \\
\hline Lesiones extuberculosas & 17 & 3,5 \\
\hline Lesiones cicatriciales no específicas & 8 & 1,7 \\
\hline Asma & 7 & 1,5 \\
\hline Aspergiloma & 7 & 1,5 \\
\hline Tuberculosis pulmonar & 4 & 1,4 \\
\hline TEP & 2 & 0,4 \\
\hline M iscelánea & 13 & 2,7 \\
\hline Idiopáticas & 15 & 3,1 \\
\hline
\end{tabular}

CPCP. Carcinoma pulmonar de células pequeñas. TEP Tromboembolismo pulmonar.

Los hallazgos y comparación de la radiografía, la TC o la FB en todos los pacientes y las etiologías más frecuentes quedan reflejados en las tablas II, III y IV. La TC no se acompañó de complicaciones y en la FB solo un caso presentó un sangrado incontrolable durante la misma con posterior fallecimiento del paciente cuya autopsia confirmó la invasión de la arteria pulmonar por un adenocarcinoma.

\section{PACIENTES CON UNA RADIOGRAFÍA DE TÓRAX NORMAL}

En 155 casos la radiografía de tórax fue considerada normal o inespecífica (32\%). La FB fue diagnóstica en el 14\% (22 casos divididos en 20 neoplasias y 2 cuerpos extraños), localizando el sangrado en 24 casos adicionales (21 por restos hemáticos localizados y 3 sangrados activos) junto a una bronquitis difusa en $86(55 \%)$ o local en $18(12 \%)$ y una exploración normal en $5(3,2 \%)$. Cuando la radiografía y la FB fueron normales (5 casos), también la TC no demostró alteraciones significativas. Cuando en la FB se confirmó una neoplasia (20 casos), en la TC objetivamos 13 masas o nódulos pulmonares, una atelectasia sin clara afectación bronquial y 6 exploraciones normales, estas últimas en un papiloma que afectaba a un bronquio subsegmentario y 5 carcinomas pulmonares de célula no pequeña ( 2 carcinomas "in situ" y 3 carcinomas epidermoides en un estadio IA). Cuando el hallazgo fue una bronquitis difusa (86 casos), la TC fue normal en 38 (44\%) o demostró la presencia de un infiltrado en 8 (9\%), bronquiectasias en 31 (36\%), un caso con una neoplasia y el resto con lesiones inespecíficas. En las bronquitis localizadas (18 casos), la TC visualizó una neoplasia en un único caso, en 10 casos bronquiectasias (56\%), lesiones residuales inespecíficas en 2 y normal en 5 casos (27\%). Ante la presencia de restos hemáticos localizados (21 casos), la TC demostró la presencia de bronquiectasias en 11 (52\%), un cuerpo extraño, un infiltrado pulmonar atribuido al sangrado, dos casos inespecíficos y fue normal en 6 casos (29\%), mientras que los tres casos con un sangrado activo endoscópico fueron diagnosticados de bronquitis crónica sin aportar una etiología la FB o la TC.

La TC fue diagnóstica en el 43\% (67 casos, 52 bronquiectasias y 15 neoplasias) junto a una localización del sangrado en 13 casos adicionales ( 3 atelectasias, 1 absceso pulmonar y 9 infiltrados) y en 62 casos fue normal (40\%). La TC fue superior a la FB en el diagnóstico etiológico (14\% FB y $43 \%$ TC, p<0,001) y la localización del sangrado ( $23 \%$ FB y $52 \%$ TC, p $<0,001)$. Cuando la TC fue considerada normal (62 casos), la FB fue normal en 12 (19\%), inespecífica en 37 (59\%, 33 con bronquitis difusa y 4 localizada) y localizó el sangrado en 12 (19\%), repartidos en 5 casos con restos hemáticos localizados, un sangrado activo y 6 con una neoplasia $(10 \%)$ ya descritas previamente. Cuando en la TC se observó una masa o nódulo pulmonar (15 casos), la FB visualizó una neoplasia en 13 (87\%) o fue inespecífica en 2 (una bronquitis difusa y una localizada), consiguiendo su confirmación histológica tras la realización de biopsias por fluoroscopia. Cuando en la TC apareció una atelectasia (3 casos), la FB confirmó casos aislados de neoplasia, cuerpo extraño y bronquitis inespecífica. Los hallazgos de la FB en los pacientes con bronquiectasias (52 casos) fueron inespecíficos (31 con una bronquitis difusa, 10 bronquitis localizadas y 11 con restos hemáticos localizados).

\section{PACIENTES CON UNA NEOPLASIA PULMONAR}

En 208 pacientes, la hemoptisis fue atribuida a una neoplasia pulmonar (43\%). La edad fue de 65,5 años (DE 11), con 12 casos de menos de 45 años (6\%), 196 varones (94\%) y 192 fumadores (92\%) de 60 paquetes/año (DE 30). El volumen de la hemoptisis fue de $17 \mathrm{ml} /$ día (DE 53) con un volumen inferior a $10 \mathrm{ml} /$ día en 169 casos $(81 \%)$ o superior a $200 \mathrm{ml} /$ día en 2 y una duración de 20 días (DE 29). 
TABLA II

RESULTADOS O BTENIDOS DE LA RADIO GRAFÍA SIM PLE DE TÓ RAX

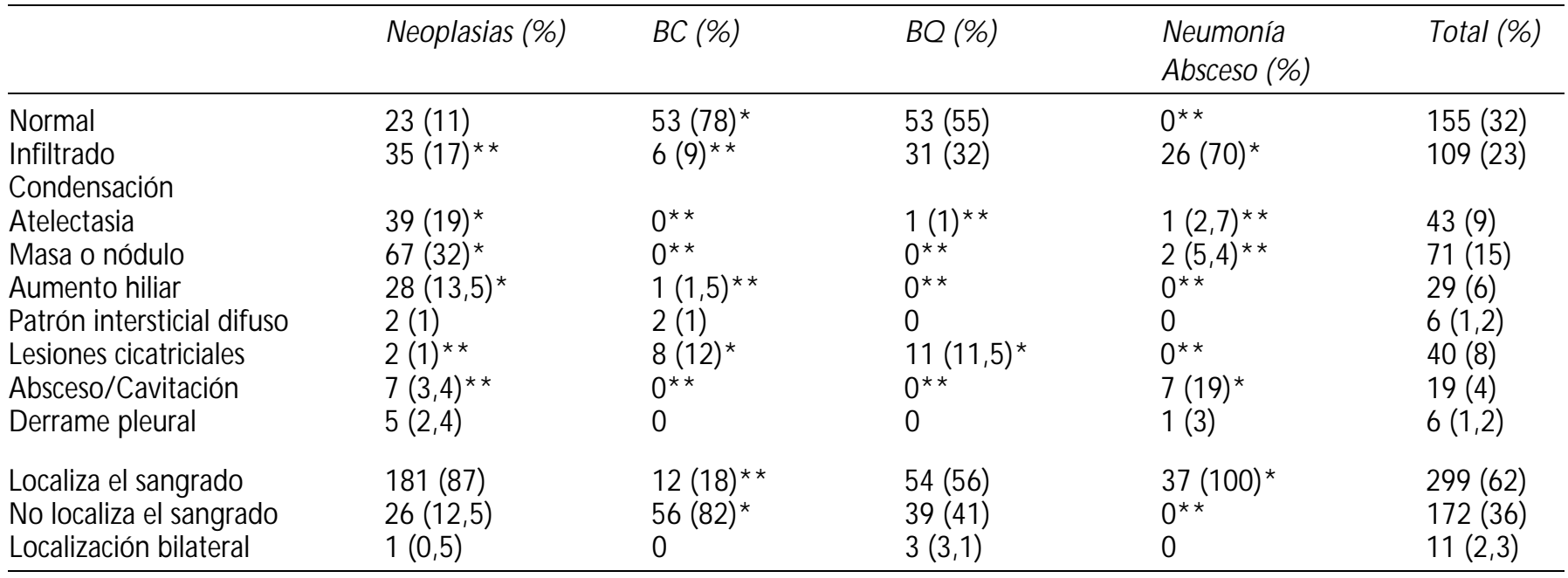

${ }^{*} p<0,05$ para los porcentajes más frecuentes. **p<0,05 para los porcentajes menos frecuentes. BC: Bronquitis crónica. BQ : Bronquiectasias.

TABLA III

RESULTADOS O BTENIDOS DE LA BRONCOSCO PIA

\begin{tabular}{llllll}
\hline & Neoplasias (\%) & BC (\%) & BQ (\%) & $\begin{array}{l}\text { Neumonía } \\
\text { Absceso }(\%)\end{array}$ & Total (\%) \\
\hline $\begin{array}{lllll}\text { Normal } \\
\text { Bronquitis difusa }\end{array}$ & $4(2)^{* *}$ & $5(7,4)^{*}$ & $0 * *$ & $4(11)^{*}$ & $18(3,8)$ \\
Bronquitis local & $20(9,6)^{* *}$ & $38(56)^{*}$ & $42(44)^{*}$ & $19(51)^{*}$ & $158(33)$ \\
$\begin{array}{l}\text { Neoplasia } \\
\text { Restos sanguíneos }\end{array}$ & $14(6,7)^{* *}$ & $6(9)^{* *}$ & $26(27)^{*}$ & $10(27)^{*}$ & $73(15)$ \\
localizados & $169(81)^{*}$ & $0 * *$ & $0 * *$ & $0 * * *$ & $169(35)$ \\
Sangrado activo & $1(0,5)^{* *}$ & $16(24)^{*}$ & $22(23)^{*}$ & $3(8,1)$ & $50(10)$ \\
Localiza el sangrado & $19(9)^{*}$ & $6(9)$ & $14(14,6)^{*}$ & $1(3)^{* *}$ & $48(10)$ \\
No localiza el sangrado & $176(84)^{*}$ & $41(60)^{* *}$ & $65(68)^{* *}$ & $21(57)^{* *}$ & $347(71,5)$ \\
Localización bilateral & $9(4,3)$ & $25(37)$ & $27(28)$ & $16(43)^{*}$ & $121(25)$ \\
\hline
\end{tabular}

${ }^{*} p<0,05$ para los porcentajes más frecuentes. ${ }^{* *} p<0,05$ para los porcentajes menos frecuentes. BC: Bronquitis crónica. BQ : Bronquiectasias

TABLA IV

RESULTADO S O BTENIDOS DE LA TC TO RÁCICA

\begin{tabular}{|c|c|c|c|c|c|}
\hline & Neoplasias (\%) & $\mathrm{BC}(\%)$ & $\mathrm{BQ}(\%)$ & $\begin{array}{l}\text { Neumonía } \\
\text { Absceso (\%) }\end{array}$ & Total (\%) \\
\hline $\begin{array}{l}\text { Normal } \\
\text { Infiltrado }\end{array}$ & $6(3)^{* *}$ & $45(66)^{*}$ & $0 * *$ & $0 * *$ & $71(15)$ \\
\hline $\begin{array}{l}\text { Condensación } \\
\text { Bronquiectasias } \\
\text { M asa o nódulo } \\
\text { Patrón intersticial difuso } \\
\text { Atelectasia } \\
\text { Cicatrices residuales } \\
\text { Absceso o cavitación }\end{array}$ & $\begin{array}{l}8(4)^{* *} \\
1(0,5)^{* *} \\
181(87)^{*} \\
1(0,5) \\
8(4) \\
1(0,5)^{* *} \\
2(1)^{* *}\end{array}$ & $\begin{array}{l}5(7,4) \\
0 * * \\
0 * * \\
0 \\
1(1,5) \\
17(25)^{*} \\
0 * *\end{array}$ & $\begin{array}{l}0 * * \\
95(99)^{*} \\
0 * * \\
0 \\
1(1) \\
0 * * \\
0 * *\end{array}$ & $\begin{array}{l}30(81)^{*} \\
0 * * \\
0 * * \\
0 \\
0 \\
0 * * \\
7(19)^{*}\end{array}$ & $\begin{array}{l}57(12) \\
96(20) \\
184(38) \\
4(0,8) \\
11(2,3) \\
39(8) \\
18(4)\end{array}$ \\
\hline $\begin{array}{l}\text { Localiza el sangrado } \\
\text { No localiza el sangrado } \\
\text { Localización bilateral }\end{array}$ & $\begin{array}{l}193(93)^{*} \\
8(3,8)^{* *} \\
7(3,4)^{* *}\end{array}$ & $\begin{array}{l}22(32)^{* *} \\
43(63)^{*} \\
3(4,4)^{* *}\end{array}$ & $\begin{array}{l}56(58) \\
0 * * \\
40(42) *\end{array}$ & $\begin{array}{l}36(97)^{*} \\
0 * * \\
1(3)^{* *}\end{array}$ & $\begin{array}{l}344(71,5) \\
80(17) \\
58(12)\end{array}$ \\
\hline
\end{tabular}


Cuando la radiografía fue normal (23 casos, 11\%), la FB aportó el diagnóstico en 20 casos (87\%) y fue inespecífica en el resto. De estos 23 casos, la TC fue normal en 6 (26\%), demostró unos hallazgos inespecíficos en 2 (una atelectasia y un infiltrado sin clara afectación bronquial) y una masa o nódulo pulmonar en 15 casos (65\%), 13 confirmados por la FB (visualización y histología) y dos por biopsias dirigidas por fluoroscopia. Cuando la radiografía de tórax fue patológica, los resultados de la FB y la TC quedaron reflejados en la tabla V. En estos casos, la FB fue superior a la radiografía en la sospecha de una neoplasia (51\% en la radiografía y $80 \%$ en la $\mathrm{FB}, \mathrm{p}<0,001$ ) a excepción de aquellos casos en que la radiografía solo visualizó una masa o nódulo pulmonar (67 casos visibles en la radiografía y 45 con una FB diagnóstica, $\mathrm{p}=0,01)$ mientras la $\mathrm{TC}$ superó a la radiografía (51\% radiografía y $88 \%$ la TC, p<0,001) o a la FB $(80 \%$ FB y $88 \%$ TC, $\mathrm{p}=0,03)$. cha de neoplasia o bronquiectasias y por un tipo de población en el que predominarían los varones fumadores de una edad superior a los 45 años en un medio en el que la tuberculosis o el uso irregular de tratamientos antibióticos en épocas anteriores provocaría la aparición posterior de sus secuelas o bronquiectasias (13-15), en las que la indicación de la TC tendría un papel fundamental a pesar de desaconsejar su uso rutinario en la mayoría de consensos por sus limitaciones económicas o pronósticas $(5,10,12)$.

La radiografía simple no garantizó un diagnóstico o la localización del sangrado en la mayoría de los casos, resultando especialmente limitada por un $32 \%$ de pacientes con una exploración normal o inespecífica que en poblaciones de riesgo como la nuestra obligarían a proseguir el estudio por la posibilidad de un diagnóstico posterior de neoplasia $(11 \%)$ o bronquiectasias $(55 \%)(6,10,16)$. Sin embargo, algunos patrones radiológicos

TABLA V

RESU LTADOS DE LA BRO NCO SCO PIA Y LA TC TORÁCICAS CUANDO LA RADIO GRAFÍA ERA ANO RM AL EN LOS PACIENTES CON UNA NEO PLASIA PULM ONAR

\begin{tabular}{|c|c|c|c|c|c|}
\hline \multicolumn{2}{|c|}{ Radiografía simple (\%) } & \multicolumn{2}{|c|}{ Broncoscopia (\%) } & \multicolumn{2}{|c|}{$\mathrm{TC}(\%)$} \\
\hline Infiltrado & $35(17)$ & $\begin{array}{l}\text { Bronquitis difusa } \\
\text { Bronquitis local } \\
\text { Neoplasia }\end{array}$ & $\begin{array}{l}4(11) \\
2(6) \\
29(83)\end{array}$ & $\begin{array}{l}\text { Infiltrado } \\
\text { M asa/Nódulo } \\
\text { Atelectasia }\end{array}$ & $\begin{array}{l}5(14) \\
29(83) \\
1(3)\end{array}$ \\
\hline Atelectasia & 39 (19) & $\begin{array}{l}\text { Bronquitis difusa } \\
\text { Neoplasia }\end{array}$ & $\begin{array}{l}1(2,6) \\
38(97,4)\end{array}$ & $\begin{array}{l}\text { Infiltrado } \\
\text { M asa/nódulo } \\
\text { Atelectasia }\end{array}$ & $\begin{array}{l}1(2,6) \\
35(90) \\
3(7,7)\end{array}$ \\
\hline M asa o nódulo & $67(32)$ & $\begin{array}{l}\text { Normal } \\
\text { Bronquitis difusa } \\
\text { Bronquitis local } \\
\text { Neoplasia } \\
\text { Restos hemáticos }\end{array}$ & $\begin{array}{l}4(6) \\
10(15) \\
7(10) \\
45(67) \\
1(1,5)\end{array}$ & $\begin{array}{l}\text { Masa/Nódulo } \\
\text { Absceso }\end{array}$ & $\begin{array}{l}66(98,5) \\
1(1,5)\end{array}$ \\
\hline Absceso o cavitación & $7(3,4)$ & $\begin{array}{l}\text { Bronquitis difusa } \\
\text { Bronquitis local } \\
\text { Neoplasia }\end{array}$ & $\begin{array}{l}3(43) \\
1(14) \\
3(43)\end{array}$ & $\begin{array}{l}\text { Masa/Nódulo } \\
\text { Absceso }\end{array}$ & $\begin{array}{l}6(86) \\
1(14)\end{array}$ \\
\hline Hilio anormal & $28(13,5)$ & Neoplasia & $28(100)$ & $\begin{array}{l}\text { M asa/Nódulo } \\
\text { Atelectasia }\end{array}$ & $\begin{array}{l}25(89) \\
3(11)\end{array}$ \\
\hline Derrame pleural & $5(2,4)$ & $\begin{array}{l}\text { Bronquitis difusa } \\
\text { Neoplasia }\end{array}$ & $\begin{array}{l}1(20) \\
4(80)\end{array}$ & $\begin{array}{l}\text { Infiltrado } \\
\text { Masa/Nódulo }\end{array}$ & $\begin{array}{l}3(60) \\
2(40)\end{array}$ \\
\hline
\end{tabular}

†Todos los casos que en la TC fueron diagnosticados por una masa o nódulo pulmonar posteriormente se confirmó que eran neoplasias. *p<0,05 para los porcentajes más frecuentes. $* * p<0,05$ para los porcentajes menos frecuentes. BC: Bronquitis crónica. BQ : Bronquiectasias.

\section{DISCUSIÓN}

La utilización conjunta de la FB y la TC en nuestra serie confirmó un mayor número de etiologías no neoplásicas destacando a las bronquiectasias, la bronquitis crónica y la neumonía o absceso pulmonar. La sistemática utilizada disminuyó el número de formas idiopáticas de hemoptisis y aumentó el porcentaje de casos atribuidos a las bronquiectasias o especialmente las neoplasias pulmonares manteniendo unos valores casi invariables en el resto de etiologías con relación a otras series recientes (12-14). Estas variaciones en la distribución etiológica estarían relacionadas con unos criterios de selección que incluirían a todos los pacientes con una sospe- fueron más frecuentes en determinadas etiologías como la presencia de una condensación o absceso pulmonar en las neumonías, una exploración normal en la bronquitis crónica y una masa pulmonar, un hilio engrosado o una atelectasia en las neoplasias, que a pesar de no ser definitivos en todos los casos, su existencia o una evolución desfavorable aconsejarían evitar una demora en la realización de la FB o la TC. La FB fue definitiva en el $81 \%$ de las neoplasias permitiendo su visualización o identificación histológica pero resultando inespecífica en la mayoría del resto de las etiologías, entre las que destacaron la presencia de una bronquitis localizada o restos hemáticos localizados cuando eran diagnosticadas de una neumonía o bronquiectasias. Si consideramos la existencia de un elevado porcentaje de etiologías 
benignas y que las imágenes endoscópicas son habitualmente insuficientes para su diagnóstico, la utilidad etiológica de la FB sería limitada y apoyaría fundamentalmente la exclusión de una neoplasia o lesión central, así como la toma de muestras para un estudio citológico y microbiológico (16-19). Nuestros hallazgos confirmaron a la TC como la técnica que aportó un mayor número de diagnósticos en nuestro medio, permitiendo el diagnóstico de todos los casos con bronquiectasias y unos patrones sugestivos de determinadas etiologías entre los que destacaron la presencia de una condensación o un absceso pulmonar en las neumonías, una masa o nódulo en las neoplasias y una exploración normal en los pacientes con bronquitis crónica, con un número limitado de casos considerados atípicos18. No obstante, la capacidad global de la TC en localizar el posible origen del sangrado fue similar a la FB, aunque algo más limitada por la presencia de lesiones bilaterales potencialmente sangrantes en la TC (12\%), que tampoco permite la visualización directa de la lesión o el sangrado, la exclusión de algunos artefactos y la realización de medidas locales de hemostasia que aconsejan realizar la FB cuando existe un mayor volumen o frecuencia de sangrado, una sospecha de varias localizaciones, existen dudas diagnósticas o interesa confirmar que el sangrado es pulmonar y el grado de afectación bronquia $(15,20,21)$.

La TC fue superior a la FB en el diagnóstico etiológico y la localización del sangrado en los pacientes con una radiografía normal o inespecífica de nuestra serie. La presencia de un mayor número de pacientes con enfermedades inflamatorias pulmonares justificaría este resultado y apoyaría la realización de la TC en nuestro medio a partir de unos criterios preestablecidos a pesar de que no se ha demostrado que su uso rutinario en todos los casos con hemoptisis tenga un gran impacto o trascendencia en su manejo $(14,15,18,22)$. Además del diagnóstico de un número elevado de bronquiectasias, su realización en una población de riesgo ayudaría a excluir otras etiologías, permitiría iniciar un tratamiento y aunque solo sea en un número limitado de casos, planear la necesidad de otras medidas si se agrava o no se controla el sangrado $(6,18,23)$. No obstante, la visualización de algunas neoplasias solo con la FB demostró que la TC aislada no fue suficiente para descartarla, manteniendo una relación complementaría que incre- menta su interés si consideramos que estas lesiones estaban en estadios iniciales y que en múltiples ocasiones la presencia de hallazgos dudosos obligan a realizar ambas exploraciones. Así, creemos que en los pacientes con una radiografía normal o inespecífica y según la distribución etiológica observada en nuestro medio, la TC debería ser la primera exploración a realizar y debería acompañarse de la FB si existe la sospecha o riesgo de una neoplasia, que podría evitarse si otro diagnóstico justificara el sangrado, el sangrado no es importante, persistente, activo, recidivante, dudoso o puede ser bilateral y responde al tratamiento $(5,6,15,18,21,22,24)$.

La neoplasia sigue siendo una de las etiologías que despierta una mayor preocupación en estos estudios y constituyó el diagnóstico etiológico aislado más frecuente en nuestra serie (43\%), sobrepasando los valores referidos por otros autores aunque justificado por la búsqueda exhaustiva e intencionada en todos los pacientes como criterio de inclusión y tratarse de un grupo de elevado riesgo como ha sido referido previamente $(7,12-14,25)$. El uso aislado de la FB, la TC y sobre todo la radiografía fueron insuficientes para excluirla en todos los casos, atribuyendo a su combinación los mejores resultados, sobre todo si la FB era incluida $(6,19,23,26)$. La FB fue la técnica más útil cuando la radiografía fue normal o inespecífica visualizando lesiones endobronquiales y permitiendo un diagnóstico definitivo en la mayoría, incluyendo aquellos en los que la TC era normal o inespecífica. La TC confirmó todos los casos que pasaron desapercibidos a la FB y viceversa, consiguiendo con su uso complementario evitar la pérdida de ningún caso después de realizar un seguimiento prolongado $(18,23,27)$. Sin embargo, cuando la radiografía fue anormal, la TC superó a la FB manteniendo su efecto complementario por la presencia de un elevado número de lesiones centrales y al establecer el diagnóstico cuando la TC era inespecífica como ocurrió ante la presencia de una atelectasia, un derrame pleural o un hilio engrosado, manteniendo unos resultados similares cuando en la radiografía aparecía un infiltrado y más limitados para la FB cuando los hallazgos en la TC o la radiografía eran una masa o absceso pulmonar habitualmente periféricos que obligaron a la utilización de la fluoroscopia o la punción transtorácica para llegar al diagnóstico definitivo.

\section{Bibliografía}

1. Cahill BC, Ingbar DH. Massive hemoptysis. Clin Chest Med 1994; 15: 147-168.

2. Roig J, Llorente JL, Ortega FJ, Orriols R, Segarra A. (Grupo de trabajo de la SEPAR). Normativa sobre el manejo de la hemoptisis amenazante. Arch Bronconeumol 1997; 33: 31-40.

3. Haro M, Jiménez J, Núñez A, Vizcaya M. Un paciente con hemoptisis. Med Integral 1999; 34: 329-336.

4. Israel RH, Poe RH. Hemoptysis. Clin Chest Med 1987; 8: 197-205.

5. Müller NL. Hemoptysis: High-resolution CT vs bronchoscopy. Chest 1994; 105: 982-983.

6. Haponik EF, Britt EJ, Smith PL, Bleecker ER. Computed chest tomography in the evaluation of hemoptysis. Impact on diagnosis and treatment. Chest 1987; 91: 80-85.

7. Hirshberg B, Biran I, Glazer M, Kramer MR. Hemoptysis: etiology, evaluation and outcome in a tertiary referral hospital. Chest 1997; 112: 440-444.

8. Dorca J, Bello S, Blanquer J, de Celis R, Molinos L, Torres A, et al. Diagnóstico y tratamiento de la neumonía adquirida en la comunidad. Arch Bronconeumol 1997; 33: 240-246.

9. Capelastegui A, Ramos J. Bronquiectasias: etiopatogenia y métodos diag-

nósticos. Arch Bronconeumol 1994; 30: 153-162.

10. Montemayor T, Alfageme Y, Escudero C, Morera J, Sánchez Agudo L. Normativa sobre el diagnóstico y tratamiento de la enfermedad pulmonar obstructiva crónica. Arch Bronconeumol 1996; 32: 285-301.

11. Casán P, Benlloch E, Duce F, Perpiñá M, Picado C, Sanchís J, Valencia A Diagnóstico del asma: lo fundamental y lo accesorio. Arch Bronconeumol 1993; 29: 1-7 (Supl.2).

12. Plaza V, Serra J, Falcó M, Brugués J. ¿Han variado las causas de hemoptisis?. Análisis de 213 casos sometidos a exploración fibrobroncoscópica. Arch Bronconeumol 1995; 31: 323-327.

13. Santiago S, Tobias J, Williams AJ. A reappraisal of the causes of hemoptysis. Arch Intern Med 1991; 151: 2449-2451.

14. McGuiness G, Beacher JR, Harkin TJ, Garay SM, Rom WN, Naidich DP Hemoptysis: prospective high-resolution CT/bronchoscopic correlation. Chest 1994; 105: 1155-1162.

15. Tasker AD, Flower CDR. Imaging the airways. Hemoptysis, bronchiectasis and small airways disease. Clin Chest Med 1999; 20: 761-773.

16. Lederle FA, Nichol KL, Parenti CM. Bronchoscopy to evaluate hemoptysis in older men with nonsuspicious chest roentgenograms. Chest 1989; 95: 1043-1047. 
17. Millar AB, Boothroyd AE, Edwards D, Hetzel MR. The role of computed tomography (CT) in yhe investigation of unexplained heamoptysis. Respir Med 1992; 86: 39-44.

18. Naidich DP, Funt S, Ettenberg NA, Arranda C. Hemoptysis: CT-bronchoscopy correlations in 58 cases. Radiology 1990; 177: 357-362.

19. Adelman M, Haponik EF, Bleecker ER, Britt EJ. Crytogenic hemoptysis. Clinical features, bronchoscopic findings and natural history in 67 patients. An Intern Med 1985; 102: 829-834.

20. Arney KL, Judson MA, Sahn SA. Airway obstruction arising from blood clot. Chest 1999; 115: 293-300.

21. Eisenhuber E, Brunner C, Bankier A. Blood clots mimicking peripheral intrabronchial tumors in patients with hemoptysis: CT and bronchoscopic findings. J Comput Assist Tomogr 2000; 24: 47-51.

22. Set PAK, Flower CDR, Smith IE, Chan AP, Twentyman OP, Shneerson
JM. Hemoptysis: comparative study of the role of CT and fiberoptic bronchoscopy. Radiology 1993; 189: 677-680.

23. Naidich DP, Harkin TJ. Airways and lung: correlation of CT with fiberoptic bronchoscopy. Radiology 1995; 197: 1-12.

24. Jackson CV, Savage PJ, Quinn DL. Role of fiberoptic bronchoscopy in patients with hemoptysis and a normal chest roentgenogram. Chest 1985; 87: 142-144.

25. Haro M, Núñez A, Vizcaya M, Tirado R, Fernández LM, Muñoz F. Importancia del sexo e influencia del tabaco en la etiología de los pacientes con hemoptisis. An Med Interna (Madrid) 1999; 16: 239-243.

26. Romero S, Martín C, Hernández L, Pronóstico a corto plazo de las hemoptisis criptogenéticas. Rev Clin Esp 1989; 185: 184-186.

27. Colice GL. Detecting lung cancer as a cause of hemoptysis in patients with a normal chest radiograph. Chest 1997; 111: 877-884. 